\author{
United Nations Educational Scientific and Cultural Organization \\ and \\ International Atomic Energy Agency \\ THE ABDUS SALAM INTERNATIONAL CENTRE FOR THEORETICAL PHYSICS
}

\title{
THERMAL STABILITY FOR A REACTIVE VISCOUS FLOW IN A SLAB
}

\author{
Samuel S. Okoya ${ }^{1}$ \\ Department of Mathematics, Obafemi Awolowo University, Ile - Ife, Nigeria \\ and \\ The Abdus Salam International Centre for Theoretical Physics, Trieste, Italy.
}

\begin{abstract}
The paper deals with the effect of dimensionless non - Newtonian coefficient on the thermal stability of a reactive viscous liquid in steady flow between parallel heated plates. It is assumed that the liquid is symmetrically heated and the flow fully developed. Approximate analytical solution is obtained for the velocity of the flow and the criterion for which this solution is valid is determined. After the velocity distribution is known, the temperature distribution may be calculated. Criticality and disappearance of criticality (transition values) are obtained in the following cases: (i) Bimolecular (ii) Arrhenius and (iii) Sensitized temperature dependence. We have observed that nonlinear effect from velocity and temperature fields introduced decaying for the transitional values of the dimensionless central temperature. Other effects of this nonlinearity are reported. We also give results for the plane - Couette flow problem. The results help to enhance understanding of the interplay between Newtonian and non - Newtonian thermal explosions.
\end{abstract}

MIRAMARE - TRIESTE

October 2002

\footnotetext{
${ }^{1}$ Regular Associate of the Abdus Salam ICTP. sokoya@oauife.edu.ng
} 


\section{Introduction}

It is well known from conductive theory of thermal explosion that the temperature equation expresses the balance between heat generated and heat conduction in terms of the standard dimensionless parameter

$$
\delta=Q E A y_{0}^{2} C_{0} k^{m} T_{0}^{m-2} \exp \left(-E / R T_{0}\right) /\left(\nu^{m} h^{m} R K\right),
$$

(see, for example, Frank - Kamenetskii [8] for the special case of $m=0$ ). Physically, $\delta$ is a reflection of the internal properties of a given system. The heat generation considers a general reaction - rate law:

$$
\begin{gathered}
K_{0}(T)=A\left(\frac{k T}{\nu h}\right)^{m} \exp \left(-\frac{E}{R T}\right)=\left[\left(\frac{k T_{0}}{\nu h}\right)^{m} \exp \left(-\frac{E}{R T_{0}}\right)\right] f(\theta ; \beta, m) \\
f(\theta ; \beta, m)=(1+\beta \theta)^{m} \exp \left(\frac{\theta}{1+\beta \theta}\right)
\end{gathered}
$$

where $\theta=\left(T-T_{0}\right) /\left(R T_{0}^{2} / E\right), \beta=R T_{0} / E$ and $m$ is a numerical exponent. Specifically, it is shown that no local steady state exists when the value of $\delta$ exceeds a critical value $\delta_{c r}$ and explosion occurs.

The natural and convenient dimensionless measure in the system are dimensionless central temperature excess $\theta_{\max }$ and the activation energy parameter $\beta$. Criticality and the disappearance of criticality have been the topic of numerous studies. In fact, a number of investigators have been able to accurately predict these transitions under physically reasonable assumptions (e.g., see Zaturska [18], Tam and Chapman [17], Boddington et al. [4, 5], Tam [16] and Okoya [10] and the references contained therein). A summary of the most important advances in this area is provided by Billingham [3] and Bowes [6]. It is worth pointing out that the critical conditions of the form described above are of obvious value in providing safety criteria both for storage and for handling.

Within the context of continuum mechanics, the heat conduction and viscous dissipation terms are of the same order of magnitude. In the event that viscous heating is not ignored, critical and transitional conditions for such a thermal mechanism can still occur. Some of the work on the effect of viscous heating on the stability of flows are as follows: Adler [1] investigated criticality for steady developed flow between symmetrically heated parallel walls when $\beta \longrightarrow 0$. Zaturska [19] studied criticality for the Poiseuille pipe flow, axial flow between concentric circular cylinders and the plane Couette flow with the same approximation on $\beta$. Shonhiwa and Zaturska [13] examined transitional values for each of the flows studied in [1] and [19] when $\beta \neq 0$. In addition, Shonhiwa and Zaturska [14] obtained transitional values for reactive plane - Poiseuille flow with approximation to the Arrhenius reaction - rate term.

In this paper, we investigate the thermal stability for a reactive viscous flow of a third grade fluid between two parallel plates with a uniform pressure gradient (plane - Poiseuille flow). 
In contrast to all earlier studies of the fluid systems for thermal explosion, our special type of flow considered here is of higher order than the corresponding Newtonian flow. After setting up the governing equations for our model problem in Section 2, we begin by studying the approximate analytical solution for the fluid velocity and the range of validity of the solution in section 3 . In section 4 , we plugged the flow velocity into the temperature equation and we obtain critical and transitional values for particular cases: the Bimolecular temperature dependence $(m=1 / 2)$ (e.g., see Boddington et al. [4, 5] and Zaturska [18]); the Arrhenius or zero - order reaction $(m=0)$ (e.g., see Bodington et al. [4, 5], Tam [16] and Tam and Chapman [17]; Sensitized temperature dependence $(m=-2)$ (e.g., see Dainton [7], Ayeni [2] and Okoya [11]); the Frank - Kamenetskii approximation $(m=0$ and $\beta<<1$ ) (e.g., see Adler [1], Frank - Kamenetskii [8], Okoya [10] and Zaturska [19]). As it stands, the Frank - Kamenetskii case grows exponentially with $\theta$. However, the Arrhenius case is bounded by $\exp (1 / \beta)$. More importantly, the Sensitized source term has a maximum of $(2 \beta)^{2} \exp ((1-2 \beta) / \beta)$ at $\theta=(1-2 \beta) / 2 \beta^{2}$, while the Bimolecular source term has a maximum of $(2 / \beta)^{1 / 2} \exp ((2-\beta) / 2 \beta)$ at $\theta=(2-\beta) / \beta^{2}$. In the remark, we also obtain similar results for the plane - Couette flow problem. Finally, in the last section a discussion and summary of the results are presented.

\section{Governing Equations}

We study the structure of the fully developed steady - state flow of an incompressible and homogeneous liquid of grade 3 under Arrhenius kinetics, confined between two infinitely long plates located at $y=-y_{0}$ and $y=y_{0}$ respectively. We seek one dimensional steady velocity fields of the form

$$
\vec{V}=u(y) \vec{i}
$$

where $\vec{i}$ denotes the unit vector in the direction of $x$ coordinate, the direction that is chosen parallel to the external pressure gradient (Figure 1):

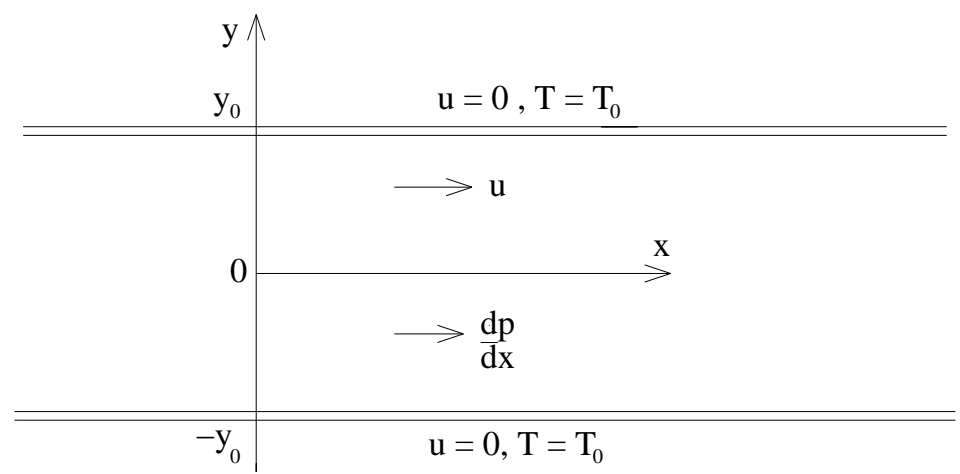

Figure 1: A schematic representation of the system under study. 
The fluid is incompressible and is hence constrained to satisfy

$$
\begin{gathered}
\operatorname{div} \vec{V}=0 \\
\frac{d^{2} u}{d y^{2}}\left(\mu+6 \beta_{3}\left(\frac{d u}{d y}\right)^{2}\right)=\frac{d p}{d x} \\
k \frac{d^{2} T}{d y^{2}}+\left(\frac{d u}{d y}\right)^{2}\left(\mu+2 \beta_{3}\left(\frac{d u}{d y}\right)^{2}\right)+Q C_{0} K_{0}(T)=0
\end{gathered}
$$

(see Szeri and Rajagopal [15] and Pai [12] for conductive and viscous terms and Boddington et al. [5] for chemical reaction term). Here $T$ is the absolute temperature, $T_{0}$ the wall temperature, $k$ the thermal conductivity of the material, $\mu$ the viscosity, $\beta_{3}$ the material coefficient, $p$ the pressure, $Q$ the heat of reaction, $A$ the rate constant, $E$ the activation energy, $R$ the universal gas constant, $C_{0}$ the initial concentration of the reactant species, $h$ the Planck's number, $\mathrm{K}$ the Boltzmann's constant, $\nu$ the vibration frequency. In this model, we neglect the consumption of the combustible material and the effect of radiant heating. We assume constant viscosity, material coefficient, thermal conductivity. It is clear from equation (2. 3) that $d p / d x$ must be a constant.

Suitable dimensionless variables are

$$
\begin{gathered}
\bar{y}=\frac{y}{y_{0}}, \quad \bar{u}=\frac{u}{U_{0}}, \quad \beta=\frac{R T_{0}}{E}, \quad \Gamma=\frac{\mu U_{0}^{2}}{k T_{0} \beta}, \\
\theta=\frac{T-T_{0}}{\beta T_{0}}, \quad \Lambda=\frac{\beta_{3} U_{0}^{2}}{\mu y_{0}^{2}}, \quad C=\frac{y_{0}^{2}}{\mu U_{0}} \frac{d p}{d x}
\end{gathered}
$$

where $U_{0}$ is a reference velocity.

The non - dimensional form of equations (2. 3) and (2.4) are

$$
\begin{gathered}
\frac{d^{2} \bar{u}}{d \bar{y}^{2}}\left(1+6 \Lambda\left(\frac{d \bar{u}}{d \bar{y}}\right)^{2}\right)=C, \\
\frac{d^{2} \theta}{d \bar{y}^{2}}+\Gamma\left(\frac{d \bar{u}}{d \bar{y}}\right)^{2}\left(1+2 \Lambda\left(\frac{d \bar{u}}{d \bar{y}}\right)^{2}\right)+\delta f(\theta ; \beta, m)=0,
\end{gathered}
$$

subject to the boundary conditions:

$$
\bar{u}(-1)=0, \quad \bar{u}(1)=0
$$

and

$$
\theta(-1)=0, \quad \theta(1)=0
$$

Here $\Gamma$ is a viscous heating parameter while $\Lambda$ is the dimensionless non - Newtonian coefficient. It is worth remarking that if $\Lambda=m=0$ we are in [13] and in addition if $\Gamma=0$ we are in [4], [5] and [6] but if only $\delta=0$ we are in [15]. In the case where $\Lambda \geq 0$, systems of the type (2. 5) and 
(2. 6) occur in many applications (see e.g. Adler [1] and Massoudi and Christie [9]). Equations (2. 5) - (2. 8) constitute a nonlinear boundary value problem for which solutions were obtained by asymptotic expansion and numerical method respectively. Solutions will be presented for three different reaction models.

\section{Asymptotic solution for the velocity}

As mentioned earlier, we are interested in examining the expansion for the velocity. The possible asymptotic expansion for the solution of (2. 5) subject to (2. 7) is

$$
\bar{u}=U_{0}+\epsilon U_{1}+\text { h. o. t. },
$$

where $\epsilon$ is the perturbation parameter and h. o. t. represents higher order terms. The ensuring analysis will be carried out on the assumption that $\Lambda$, the dimensionless non - Newtonian coefficient is small compared to Newtonian term. Thus

$$
\Lambda=\epsilon \lambda+\text { h. o. t.. }
$$

The boundary value problems which uniquely determine the coefficients of the expansion (3. 1) subject to (3. 2) are listed in the following order:

$$
\begin{aligned}
& \bigcirc(1): U_{0}^{/ /}=C ; U_{0}(-1)=U_{0}(1)=0, \\
& \bigcirc(\epsilon): U_{1}^{/ /}+6 \lambda U_{0}^{/ /}\left(U_{0}^{/}\right)^{2}=0 ; U_{1}(-1)=U_{1}(1)=0,
\end{aligned}
$$

where prime denotes derivative with respect to $\bar{y}$. From simple integration of equations (3. 3) and (3.4) we obtain

$$
U_{0}=\frac{C}{2}\left(\bar{y}^{2}-1\right) \text { and } U_{1}=-\frac{\Lambda C^{3}}{2}\left(\bar{y}^{4}-1\right),
$$

Substituting (3. 5) into the expansion in (3. 1), returning back to the original parameter since $\epsilon$ is artificially introduced, one has

$$
\bar{u}=\frac{C}{2}\left(\bar{y}^{2}-1\right)-\frac{\Lambda C^{3}}{2}\left(\bar{y}^{4}-1\right) .
$$

The second terms are the correction terms in the perturbation expansion and hence should be much smaller than preceding terms to assure the validity of the expansion. Hence in absolute values

$$
\frac{\Lambda C^{3}}{2} \ll \frac{C}{2} \quad\left(\text { or } \Lambda C^{2} \ll 1\right)
$$

We now contrast the perturbation solution with numerical solution obtained by Maple. 


$\begin{array}{cccc}\mathrm{C} & \bar{u}_{\max } & \bar{u}_{\max } & \Lambda C^{2} \\ (\text { Pert.) } & \text { (Maple) } & \\ -0.25 & 0.1234 & 0.1235 & 0.0125 \\ -0.50 & 0.2375 & 0.2394 & 0.0500 \\ -0.75 & 0.3328 & 0.3446 & 0.1125 \\ -1 & 0.4000 & 0.4390 & 0.2000 \\ -2 & 0.2000 & 0.7374 & 0.8000 \\ -3 & -1.200 & 0.9572 & 1.8000 \\ -4 & -4.400 & 1.1133 & 3.2000 \\ -5 & -10.00 & 1.2807 & 5.0000\end{array}$

Table 1: Comparison of perturbation solution and numerical result when $\Lambda=0.20$.

For $|C| \geq 1$ with $\Lambda=0.20$ in Table 1 , the criterion becomes large and so the results are not in agreement with numerical results. For $|C|<1$, results are in good agreement. Take $|C|=0.25$. In Table 2, variation of $\Lambda$, the dimensionless non - Newtonian coefficient, is considered. For $|\Lambda| \geq 2$, the non - Newtonian criterion is violated and the results do not match with the Maple. For $\Lambda=0$, results are the same while for $\Lambda \leq 1$ the results are the same to one decimal place.

$\begin{array}{cccc}\Lambda & \bar{u}_{\max } & \bar{u}_{\max } & \Lambda C^{2} \\ (\text { Pert. } & (\text { Maple }) & \\ 0.00 & 0.1250 & 0.1250 & 0.0000 \\ 0.25 & 0.1211 & 0.1231 & 0.0156 \\ 0.50 & 0.1172 & 0.1215 & 0.0312 \\ 1 & 0.1094 & 0.1186 & 0.0625 \\ 2 & 0.0938 & 0.1140 & 0.1250 \\ 3 & 0.0781 & 0.1104 & 0.1875 \\ 4 & 0.0625 & 0.1074 & 0.2500 \\ 5 & 0.0469 & 0.1048 & 0.3125 \\ 10 & -0.0312 & 0.0957 & 0.6250\end{array}$

Table 2: Comparison of perturbation solution and numerical result when $\mathrm{C}=-0.25$.

Now that the velocity of motion has been evaluated, we turn to the energy equation.

\section{Energy equation}

The overall energy balance may be reduced exactly to the form

$$
\frac{d^{2} \theta}{d \bar{y}^{2}}+\delta(1+\beta \theta)^{m} \exp \left(\frac{\theta}{1+\beta \theta}\right)+\Gamma g(\bar{y})=0
$$

subject to

$$
\theta(-1)=0, \quad \theta(1)=0
$$

where

$$
g(\bar{y})=\left(C \bar{y}-2 \Lambda C^{3} \bar{y}^{3}\right)^{2}\left(1+2 \Lambda\left(C \bar{y}-2 \Lambda C^{3} \bar{y}^{3}\right)^{2}\right)
$$


Because there is no general analytical solution for equations (4. 1) - (4. 3) as well as the fact that $\theta(\bar{y})$ at the point of explosion is not stable, numerical methods are required to determine the critical and the transition values of the parameters $\beta, \delta$ and $\theta_{\max }$.

For solving equations (4. 1) and (4. 2) for criticality, a numerical procedure in Maple based on a shooting method with unknown parameter was applied with the unknown parameter taken to be the Frank - Kamenetskii number $\delta$ for fixed $\beta=0.05$. Criticality corresponds to a turning point in the curve of $\delta$ versus $\theta_{\max }$ while criticality ceases to exist when $\delta$ becomes a monotonic increasing function of $\theta_{\max }$. In the event that $\beta \ll 1$ (Frank - Kamenetskii approximation) there is no transition. In order to test our numerical method we first solve the Frank Kamenetskii problem by setting $\beta=\Gamma=0$. This is in agreement with Boddington et al. [4, 5]. Our numerical results for transition when $\Lambda=m=0$ and $\Gamma=0, m=1 / 2$ shows consistency with Shonhiwa and Zaturska [19] and Boddington et al. [4, 5] respectively. We now present results of the variation of the critical parameters with respect to the non - Newtonian coefficient $\Lambda$ in Tables 3 and 4 . In addition, Table 4 shows the variation of the critical parameters with respect to the exponent $m$.

\begin{tabular}{lcccccc} 
& \multicolumn{2}{c}{$\Lambda=0$} & \multicolumn{2}{c}{$\Lambda=0.1$} & \multicolumn{2}{c}{$\Lambda=0.2$} \\
$\Gamma$ & $\delta_{c r}$ & $\theta_{\text {max }, c r}$ & $\delta_{c r}$ & $\theta_{\text {max }, c r}$ & $\delta_{c r}$ & $\theta_{\text {max }, c r}$ \\
0.0 & 0.878458 & 1.186842 & 0.878458 & 1.186842 & 0.878458 & 1.186842 \\
1.6 & 0.871521 & 1.195013 & 0.871557 & 1.194971 & 0.871595 & 1.194927 \\
16 & 0.811473 & 1.268568 & 0.811810 & 1.268149 & 0.812160 & 1.267713 \\
160 & 0.396087 & 2.005913 & 0.397744 & 2.001702 & 0.399470 & 1.997332 \\
800 & 0.015610 & 5.306334 & 0.015942 & 5.285154 & 0.016295 & 5.263177
\end{tabular}

Table 3. Criticality under exponential approximation.

Furthermore, we showed in Table 5 the variation of transitional parameters with respect to the non - Newtonian parameter $\Lambda$ and the exponent $m$. 


\begin{tabular}{ccccccc}
\multicolumn{7}{c}{$\Gamma=0$} \\
$\mathrm{~m}$ & $\delta_{c r} \Lambda=0$ & $\theta_{\max , c r}$ & $\delta_{c r}$ & $\theta_{\text {max }, c r}$ & $\delta_{c r}$ & $\theta_{\text {max }, c r}$ \\
0.5 & 0.903816 & 1.290899 & 0.903816 & 1.290899 & 0.903816 & 1.290899 \\
0.0 & 0.928400 & 1.329203 & 0.928400 & 1.329203 & 0.928400 & 1.329203 \\
-2 & 1.042336 & 1.510163 & 1.042336 & 1.510163 & 1.042336 & 1.510163 \\
& & & $\Gamma=1.6$ & & & \\
0.5 & 0.897238 & 1.300143 & 0.897272 & 1.300095 & 0.897307 & 1.300456 \\
0.0 & 0.921836 & 1.338496 & 0.921870 & 1.338449 & 0.921906 & 1.338399 \\
-2 & 1.035843 & 1.519708 & 1.035877 & 1.519659 & 1.035912 & 1.519608 \\
& & & $\Gamma=16$ & & & \\
0.5 & 0.840344 & 1.383382 & 0.840663 & 1.382906 & 0.840995 & 1.382412 \\
0.0 & 0.865013 & 1.422187 & 0.865332 & 1.421708 & 0.865663 & 1.421212 \\
-2 & 0.979424 & 1.605676 & 0.979742 & 1.605184 & 0.980072 & 1.604673 \\
& & & $\Gamma=160$ & & & \\
0.5 & 0.446761 & 2.219980 & 0.448349 & 2.215183 & 0.450003 & 2.210204 \\
0.0 & 0.468540 & 2.263480 & 0.470154 & 2.258654 & 0.471836 & 2.253646 \\
-2 & 0.572031 & 2.470864 & 0.573754 & 2.465893 & 0.575550 & 2.460734 \\
& & & $\Gamma=800$ & & & \\
0.5 & 0.042489 & 6.010139 & 0.043054 & 5.985524 & 0.043649 & 5.959986 \\
0.0 & 0.048148 & 6.079638 & 0.048764 & 6.054824 & 0.049414 & 6.029078 \\
-2 & 0.080401 & 6.424375 & 0.081276 & 6.398469 & 0.082196 & 6.371593
\end{tabular}

Table 4: Criticality under Bimolecular, Arrhenius and Sensitized reaction.

\begin{tabular}{|c|c|c|c|c|c|c|c|c|c|}
\hline \multirow[b]{2}{*}{$\mathrm{m}$} & & $\Lambda=0.0$ & & & $\begin{array}{c}\Gamma=0 \\
\Lambda=0.1\end{array}$ & & & $\Lambda=0.2$ & \\
\hline & $\beta_{t r}$ & $\delta_{t r}$ & $\theta_{\max , t r}$ & $\beta_{t r}$ & $\delta_{t r}$ & $\theta_{\max , t r}$ & & & \\
\hline 0.5 & 0.33692 & 1.16777 & 5.10199 & 0.33692 & 1.16777 & 5.10199 & 0.33692 & 1.16777 & 5.10199 \\
\hline 0.0 & 0.24578 & 1.30737 & 4.89655 & 0.24578 & 1.30737 & 4.89655 & 0.24578 & 1.30737 & 4.89655 \\
\hline-2 & 0.13215 & 1.67153 & 5.19224 & 0.13215 & $\begin{array}{c}1.67153 \\
\Gamma=1.6\end{array}$ & 5.19224 & 0.13215 & 1.67153 & 5.19224 \\
\hline 0.5 & 0.33603 & 1.16330 & 5.13665 & 0.33604 & 1.16332 & 5.13649 & 0.33604 & 1.16335 & 5.13616 \\
\hline 0.0 & 0.24531 & 1.30220 & 4.92376 & 0.24531 & 1.30223 & 4.92327 & 0.24531 & 1.30225 & 4.92291 \\
\hline-2 & 0.13201 & 1.66537 & 5.21099 & 0.13201 & $\begin{array}{c}1.66540 \\
\Gamma=16\end{array}$ & 5.21079 & 0.13201 & 1.66544 & 5.21058 \\
\hline 0.5 & 0.32846 & 1.12419 & 5.45179 & 0.32851 & 1.12441 & 5.44918 & 0.32855 & 1.12464 & 5.44871 \\
\hline 0.0 & 0.24121 & 1.25698 & 5.16718 & 0.24123 & 1.25721 & 5.16560 & 0.24125 & 1.25748 & 5.16384 \\
\hline-2 & 0.13080 & 1.61115 & 5.38223 & 0.13080 & $\begin{array}{l}1.61146 \\
\Gamma=160\end{array}$ & 5.38130 & 0.13081 & 1.61178 & 5.37976 \\
\hline 0.5 & 0.27680 & 0.82440 & & 0.27702 & 0.82579 & & 0.27725 & 0.82723 & 8.37092 \\
\hline 0.0 & 0.21078 & 0.90647 & 7.49070 & 0.21092 & 0.90810 & 7.47795 & 0.21106 & 0.90979 & 7.46418 \\
\hline-2 & 0.12062 & 1.17171 & 7.05337 & 0.12067 & $\begin{array}{l}1.17382 \\
\Gamma=800\end{array}$ & 7.04430 & 0.12073 & 1.17601 & 7.03385 \\
\hline 0.5 & 0.19195 & 0.28986 & 19.96715 & 0.19225 & 0.29156 & 19.89595 & 0.19257 & 0.29335 & 19.82358 \\
\hline 0.0 & 0.15311 & 0.28886 & 16.79962 & 0.15333 & 0.29075 & 16.74222 & 0.15356 & 0.29272 & 16.68138 \\
\hline-2 & 0.09571 & 0.34383 & 14.03324 & 0.09582 & 0.34635 & 13.97942 & 0.09593 & 0.34899 & 13.93365 \\
\hline
\end{tabular}

Table 5: Transitional values for Bimolecular, Arrhenius and Sensitized reaction. 


\section{Remark}

In the second instance we allow $d p / d x=0$. The lower wall is at rest and the upper is in uniform motion ( say $u=u_{0} \neq 0$ ). This corresponds to the plane Couette flow. In this special case, equation (2.5) reduces to

$$
\frac{d^{2} \bar{u}}{d \bar{y}^{2}}\left(1+6 \Lambda\left(\frac{d \bar{u}}{d \bar{y}}\right)^{2}\right)=0
$$

subject to the boundary conditions

$$
\bar{u}(-1)=0, \quad \bar{u}(1)=1,
$$

where $\bar{u}=u / u_{0}$. The solution is seen to be given by

$$
\bar{u}=(\bar{y}+1) / 2 .
$$

In this case, the non - Newtonian parameter $\Lambda$ does not affect the flow in any sense. Substitute equation (iii) in equation (2. 6), we have

$$
\frac{d^{2} \theta}{d \bar{y}^{2}}+\delta(1+\beta \theta)^{m} \exp \left(\frac{\theta}{1+\beta \theta}\right)+\frac{\Gamma}{4}=0 .
$$

Tables 6 and 7 give our results for criticality of equation $(i v)$ subject to the boundary conditions

\begin{tabular}{|c|c|c|c|c|c|c|}
\hline \multirow[b]{2}{*}{$\mathrm{m}$} & \multicolumn{2}{|c|}{$\Gamma=0$} & \multicolumn{2}{|c|}{$\Gamma=0.4$} & \multicolumn{2}{|c|}{$\Gamma=4$} \\
\hline & $\delta_{c r}$ & $\theta_{\max , c r}$ & $\delta_{c r}$ & $\theta_{\max , c r}$ & $\delta_{c r}$ & $\theta_{\max , c r}$ \\
\hline 0.5 & 0.903816 & 1.290899 & 0.868795 & 1.345235 & 0.611936 & 1.836363 \\
\hline 0.0 & 0.928400 & 1.329203 & 0.893445 & 1.383814 & 0.635818 & 1.877457 \\
\hline \multirow[t]{2}{*}{-2} & 1.042336 & 1.510163 & 1.007719 & 1.566155 & 0.747599 & 2.072560 \\
\hline & \multicolumn{2}{|c|}{$\Gamma=20$} & \multicolumn{2}{|c|}{$\Gamma=80$} & \multicolumn{2}{|c|}{$\Gamma=200$} \\
\hline $\mathrm{m}$ & $\delta_{c r}$ & $\theta_{\max , c r}$ & $\delta_{c r}$ & $\theta_{\max , c r}$ & $\delta_{c r}$ & $\theta_{\max , c r}$ \\
\hline & 0.145797 & 4.055470 & 0.003206 & 12.711276 & 0.000072 & 31.343701 \\
\hline 0.0 & 0.158542 & 4.109157 & 0.004047 & 12.841943 & 0.000114 & 31.862591 \\
\hline-2 & 0.223959 & 4.369557 & 0.010464 & 13.537658 & 0.000737 & 35.607956 \\
\hline
\end{tabular}
(4. 2).

$\begin{array}{ccccccc}\Gamma & 0.0 & 0.4 & 4 & 20 & 80 & 200 \\ \delta_{c r} & 0.878458 & 0.841440 & 0.569182 & 0.094558 & 0.000078 & 3.40701(-11) \\ \theta_{\text {max }, \text { cr }} & 1.186842 & 1.235279 & 1.672226 & 3.631242 & 11.075664 & 26.048217\end{array}$

Table 6. Criticality under exponential approximation.

Table 7: Criticality under Bimolecular, Arrhenius and Sensitized reaction.

We now consider computed results for transition (loss of criticality) for $m$ in Table 8 . 


$\begin{array}{cccc} & & \Gamma=0 & \\ \mathrm{~m} & \beta_{t r} & \delta_{t r} & \theta_{\text {max }, t r} \\ 0.5 & 0.33692 & 1.16777 & 5.10199 \\ 0.0 & 0.24578 & 1.30737 & 4.89655 \\ -2 & 0.13215 & 1.67153 & 5.19224 \\ & & \Gamma=0.4 & \\ 0.5 & 0.33228 & 1.14422 & 5.29692 \\ 0.0 & 0.24328 & 1.28008 & 5.04841 \\ -2 & 0.13141 & 1.63881 & 5.30008 \\ & & \Gamma=4 & \\ 0.5 & 0.29926 & 0.96207 & 6.98862 \\ 0.0 & 0.22443 & 1.06744 & 6.38103 \\ -2 & 0.12540 & 1.37648 & 6.26009 \\ & & \Gamma=20 & \\ 0.5 & 0.22751 & 0.50809 & 13.83416 \\ 0.0 & 0.17808 & 0.53516 & 11.89654 \\ -2 & 0.10725 & 0.67410 & 10.37614 \\ & & \Gamma=80 & \\ 0.5 & 0.14870 & 0.09415 & 36.92938 \\ 0.0 & 0.12054 & 0.08084 & 30.66711 \\ -2 & 0.07830 & 0.07706 & 24.88957 \\ & & \Gamma=200 & \\ 0.5 & 0.10434 & 0.00921 & 80.25541 \\ 0.0 & 0.08590 & 0.00565 & 66.28153 \\ -2 & 0.05789 & 0.00290 & 52.40111\end{array}$

Table 8: Transitional values for Bimolecular, Arrhenius and Sensitized reaction.

\section{Conclusion}

Ignition phenomena for plane - Poiseuille flow between parallel plates at a distance $2 \overline{y_{0}}$ apart and held at temperature $T_{0}$ is studied, where the effects of non - Newtonian coefficient $\Lambda$ and the exponent $m$ are both accounted for. The resulting coupled equations for the plane Poiseuille flow are stated. The important features of these systems are compactly represented in dimensionless terms. The velocity equation is solved by perturbation method and compared with Maple results while the temperature equation is solved numerically to study the criticality and transitional values of the parameters that are used in the model. The major observations and conclusions are as follows:

Both $\delta_{c r}$ and $\theta_{\max , c r}$ are monotonically increasing functions of the non - Newtonian coefficient. In addition, both $\delta_{c r}$ and $\theta_{\max , c r}$ increase as $m$ decreases. Furthermore, $\beta_{t r}$ is a monotonically increasing function of the exponent $m$, while the parameter $\delta_{t r}$ is a monotonically decreasing function. The case of $\theta_{\max , t r}$ is of two fold. For small values of $\Gamma, \theta_{\max , t r}$ is a concave function of the exponent $m$ (e.g., see in particular the first to the third block of the results in Table 5) while for large values of $\Gamma$, it is monotonically increasing (see in particular the last two blocks of the results in Table 5). 
We observe from the remark that the non - Newtonian coefficient does not affect the velocity in the case of the plane Couette flow. Furthermore, like in the preceding paragraph, $\delta_{c r}$ and $\theta_{\max , c r}$ are decreasing functions of the exponent $m . \beta_{t r}$ is a monotonically increasing function of the exponent $m$, while the parameter $\delta_{t r}$ is a monotonically decreasing function. $\theta_{\text {max,tr }}$ is also of the same two fold; for small values of $\Gamma$, it is a concave function of the exponent $m$ (see in particular the first to the third block of the results in Table 8), while for large values of $\Gamma, \theta_{\max , t r}$ is monotonically increasing (e.g., see in particular the last three blocks of the results in Table 8). These scenarios of $\theta_{\max , t r}$ might not be unconnected with the fact that circumstances normally described by thermal explosion theory indicates that $\Gamma$ is small. Values of $\Gamma$ around 800 for plane - Poiseuille flow (or 200 for plane - Couette flow) are not usual but are included for completeness.

It is observed that where there is common ground with earlier results agreement is excellent.

Finally, the case of infinitely long pipe of circular cross section, axial flow between circular cylinder and flow between differentially rotating concentric cylinders are the subject of an on going work. When the five reactive flows are inclined and /or viscosity is accounted for are still open problems.

\section{Acknowledgments}

I gratefully acknowledge the financial support of the Swedish International Development Cooperation Agency (SIDA) to visit the Abdus Salam International Centre for Theoretical Physics (ICTP), Trieste, Italy where this research work was done and the paper was written. The kind hospitality of the Abdus Salam ICTP is also gratefully acknowledged. 


\section{References}

1. J. Adler, Thermal explosion theory for reactive flow between parallel heated walls. Combustion and Flame, 24 (2), 151 - 158, 1975.

2. R. O. Ayeni, On the explosion of chain - thermal reactions. J. Austral Math. Soc. B, 24, $194-202,1982$.

3. J. Billingham, Steady - state solutions for strongly exothermic ignition in symmetric geometries. IMA Journal of Applied Mathematics, 65, 283 - 313, 2000.

4. T. Boddington, C. G. Feng and P. Gray, Thermal explosions, criticality and the disappearance of criticality in systems with distributed temperatures. I. Arbitrary Biot number and general reaction - rate laws. Proc. R. Soc. Lond., A390, 247 - 264, 1983.

5. T. Boddington, C. G. Feng and P. Gray, Thermal explosions, criticality and the disappearance of criticality in systems with distributed temperatures. II. An asymptotic analysis of criticality at the extremes of Biot number $((B i) \rightarrow 0,(B i) \rightarrow \infty)$ for general reaction rate- laws. Proc. R. Soc. Lond., A392, $301-322,1984$.

6. P. C. Bowes, Self heating: Evaluating and controlling the hazards. Amsterdam: Elsevier Science, New York, 1984.

7. F. S. Dainton, Chain reactions: an introduction. $1^{\text {st }}$ ed., Wiley, New York, 1966.

8. D. A. Frank - Kamenetskii, Diffusion and heat transfer in chemical kinetics. $2^{\text {nd }}$ ed., New York, Plenum Press, 1969.

9. M. Massoudi and I. Christie, Effects of variable viscosity and viscous dissipation on the flow of a third grade fluid in a pipe. Int. J. Non - Linear Mechanics, Vol. 30, No. 5, 687 699, 1995.

10. S. S. Okoya and S. O. Ajadi, Critical parameters for thermal conduction equations. Mechanics Research Communications. Vol. 26, No. 3, 363 - 370, 1999.

11. S. S. Okoya, On the behaviour of solutions to a system of ordinary differential equations modelling branched - chain reaction. International Communication in Heat and Mass Transfer. Accepted, 2002.

12. Shih - I Pai, Viscous flow theory 1 - Laminar flow. D. Van Norstrand Company, New Jersey. U. S. A., 1956.

13. T. Shonhiwa and M. B. Zaturska, Disappearance of criticality in thermal explosion for reactive viscous flows.Combustion and Flame, 67, 175 - 177, 1987. 
14. T. Shonhiwa and M. B. Zaturska, Disappearance of criticality in thermal ignition for a simple reactive flow. Z. Angew. Math. Phys., 37, 632 - 635, 1986.

15. A. Z. Szeri and K. R. Rajagopal, Flow of a Non - Newtonian fluid between heated parallel plates. Int. J. Non - Linear Mechanics, Vol. 20, No. 2, 91 - 101, 1985.

16. K. K. Tam, On the disappearance of criticality in the theory of thermal ignition. Z. Angew. Math. Phys., Vol 31, No. 6, 762 - 766, 1980.

17. K. K. Tam and P. B. Chapman, Thermal ignition in a reactive slab with unsymmetric boundary temperatures. J. Austral. Math. Soc. Ser. B, Vol. 24, No. 3, 279 - 288, $1982 / 83$.

18. M. B. Zaturska, Approximations in thermal explosion theory and the nature of the degenerate critical point. Z. Angew. Math. Phys., Vol. 33, No. 3, 379 - 391. 1982.

19. M. B. Zaturska, Critical conditions for thermal explosion in reactive viscous flows. Combustion and Flame, 41, 201 - 211, 1981. 